\title{
Exposición critica sobre las perspectivas teóricas de la Antropología cognitiva
}

\author{
Marcelo Arnold
}

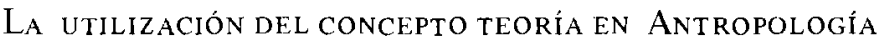

La perspectiva cognitiva ha sido reconocida como una de las más modernas versiones teóricas de que dispone la Antropología sociocultural. A pesar de ello, el nivel estrictamente teórico de que dispone y en el cual se apoyan sus análisis y explicaciones de fenómenos culturales es discutible, como también lo ha sido la cientificidad de las perspectivas que lo anteceden o que compiten con ella. Creemos que la raíz de este problema se encuentra en la misma utilización del concepto de teoría en Antropología.

La aplicación del concepto de teoría en Antropología es bastante difusa y no es discriminatoria de las diferentes acepciones de que dispone ese concepto, lo cual contribuye a la indeterminación de sus alcances. Es habitual participar en supuestos debates teóricos, pero es bastante infrecuente la delimitación del concepto de teoría y la pertinencia de aludir a aquel nivel del conocimiento cuando son debatidas cuestiones que no van más allá de las meras conjeturas 0 , en el mejor de los casos, de hipótesis inconexas.

En el plano local, a menudo, se alude a unas supuestas carencias en la formación teórica de los estudiantes y a la consecuente necesidad de implementar cursos de teoría. En otro sentido, es corriente que se califiquen algunas teorías como pasadas de moda y obsoletas, y muchos se quejan de estar al margen de las modernas teorías antropológicas. Al parecer, el conocimiento de la teoría es vital para el antropólogo y su formación, pero permanece la interrogante: ¿Qué entendemos los antropólogos por teoría? ${ }^{1}$.

\footnotetext{
${ }^{1}$ Por cierto, el ambiente de esta discusión se complica más ante la presencia de los autodenominados conocedores de las últimas teorias y que de paso descartan toda discusión al respecto, o de aquellos que manejan los conceptos de teoría y de ideología como sinónimos, desencadenando con ello una confusión sin límites.
} 
Siendo nuestra materia tan confusa y polémica no podemos eludirla, en vista de lo cual nos permitiremos exponer y someter a discusión nuestras propias ideas acerca de la controvertida existencia de la teoría antropológica. No hay nada más práctico para el desarrollo de la Antropología que disponer de buenas y modernas teorías; sin embargo, creemos que muchas veces la búsqueda de novedades que potencian nuestras ideas acerca de los fenómenos culturales debe ser dirigida hacia una relectura de nuestras obras clásicas, a un análisis de nuestras propias tradiciones de pensamiento y no desgastarse tanto en estériles modas intelectuales.

Gran parte del problema que gira en torno a la teoría en nuestra disciplina se debe a que automáticamente, cuando discutimos en los ambientes académicos. tenemos en mente un imperfecto modelo acerca de las ciencias naturales y especialmente de la Física. Estas ciencias, dado su probado nivel de análisis y capacidad de explicación y de predicción son consideradas como un ideal, esto es, como una imagen paradigmática para las ciencias sociales. Los éxitos y desaciertos de estas últimas son incluso evaluadas con ese patrón. Por cierto, muchos antropólogos aspiran hacer de su ciencia algo parecido a lo ocurrido con las ciencias de la naturaleza, pero, a pesar de los esfuerzos por alcanzar esa meta, sus logros han sido más bien escasos y modestos.

Si nuestra concepción de teoría se modela, por ejemplo, a partir de las ciencias físicas, no hay duda alguna de que las ciencias sociales, y con ellas la Antropología, están en una fase absolutamente preteórica. Al respecto es interesante e ilustrativo señalar algunas observaciones que ha hecho sobre estas materias el sociólogo R. Merton: "... un número muy elevado de estudios sobre el método científico han formulado los requisitos previos lógicos de la teoría científica, pero, al parecer, con frecuencia lo han hecho a un nivel de abstracción tan elevado que la perspectiva de traducir esos preceptos a la investigación sociológica [y antropológica] actual resulta utópica" (p. 95).

Cuando los antropólogos D. Kaplan y R. Manners discurren acerca de las causas del desnivel entre las que llamamos teorías en Antropología con respecto de las existentes en las ciencias naturales, se preguntan: "¿Por qué la Antropología no ha producido las elegantes teorías deductivas que admiran los filósofos? [...] ¿Se trata de un problema de madurez de la Antropología o es un problema inherente al tipo de dato con que trabajamos?" (1968, p. 9). Al comparar los logros de disciplinas tan disímiles, estos autores sugieren tomar en cuenta previamente algunas consideraciones. Por ejemplo, determinar comparativamente la complejidad de uno y otro objeto de estudio, esto es, reconocer la diferencia de complejidad que existe entre los fenómenos naturales y los fenómenos socioculturales; superar la dificultad que se asocia al estudio de sistemas históricos, es decir, abiertos al cambio y a la contingencia y, por último, explorar y explicitar la continua presencia de factores extracientíficos en la 
construcción de teorías sobre la sociedad y la cultura, en especial referencia al papel que cumplen las ideologías - políticas y religiosasen la formulación de ellas al interior de las ciencias sociales y humanas.

No deja de ser curioso. y a la vez estimulante, que el reiterado reconocimiento de nuestras dificultades en materia de construcción de teorías no se haya constituido en una limitación. corno para que autores rigurosos, como los antes aludidos, compilen un texto y lo titulen ostentosamente Theory in Anthropology: a source-book, lo que no es por cierto ninguna excepción: otros libros, igualmente muy leídos, tienen títulos y temáticas similares, por ejemplo, el del polémico Marvin Harris, The rise of the anthropological theory (1968), o el de Rossi y O'Higgins, Theories of Culture and Anthropological Methods (1980). Pero, en el fondo, ¿a qué se están refiriendo estos autores cuando escriben acerca de las teorías antropológicas? Esta interrogante pretendemos dilucidarla a continuación, aunque, por cierto, no intentamos ni remotamente dar cuenta final de esa problemática; el ánimo que nos guia es el de estimular una discusión consciente a nivel nacional.

En un articulo muy esclarecedor, Merton, hace algunos años, disínguió, con una precisión aún no superada, seis tipos de trabajos que son frecuentemente englobados bajo el concepto de teoría en las ciencias sociales: los que atañen a metodologías; orientaciones generales que comprenden amplios postulados y que indican tipos de variables que hay que tomar en cuenta, pero que no especifican la dirección de sus relaciones; análisis de conceptos aislados; ilustraciones a partir de interpretaciones post factum; generalizaciones empíricas ausentes de explicación y, por último, teorías propiamente tales (pp. 97-105). Complementando lo anterior, Kaplan y Manners (1981), ante las nuevas tendencias, han insistido vigorosamente en distinguir entre los modelos, es decir, ciertas formas de reduccionismo, medios para reducir y simplificar los complejos fenómenos socioculturales y, por tanto, recursos heurísticos no explicativos y las teorías propiamente tales cuya función es explicativa. A pesar de todo lo ya conocido, el mismo Merton, descorazonadamente, como tantos otros autores, sólo discute los criterios lógicos para la construcción de teorías, pero no encuentra ningún ejemplo que satisfaga esos criterios.

Como las rutinas dan lugar a las costumbres y éstas a las instituciones, incluida la que denominamos actividad científica, podemos, a la luz del análisis del uso común que se aplica al concepto de teoría en la Antropología, caracterizar la actividad teórica en términos de un cuerpo bastante difuso, resultante de la reflexión intelectual aplicada sobre materiales empíricos, reunidos - por lo general-de una manera sistemática y acorde con las convenciones científicas vigentes, del cual se derivan ideas y conceptos que facilitan --a la vez que constituyen un nexo-- el reconocimiento y explicación de fenómenos socioculturales. 
Debe recalcarse que para el caso de la Antropología, los problemas inherentes a la construcción de teorías son aún más complejos que en otras disciplinas humanas. Nuestra ciencia está fuertemente influida por concepciones relativistas que inhiben y cuestionan las comparaciones interculturales que darían lugar para la generación de cuerpos de hipótesis y de teorías de amplio alcance acerca de la cultura y al descubrimiento de relaciones de causalidad -o probabilísticas- de carácter universal, tal como lo esbozaron los evolucionistas clásicos. La fuerte y demoledora crítica de Franz Boas contra las pretensiones de los evolucionistas, los cuales a partir de procedimientos comparativos propugnaban la búsqueda de leyes generales aplicables a la evolución sociocultural, es el origen de una confrontación que llega hasta nuestros días entre los enfoques nomotéticos y los particularistas en Antropología. La relativización boasiana se apoya en un importante argumento: elementos culturales similares pueden originarse en diferentes culturas sobre la base de causas muy disímiles.

Ante el reconocimiento de sus limitaciones, la actividad teórica, en el campo de la Ciencias Scciales, ha sido denominada de muy diversas maneras: orientación sociologica general (MERTON, R.); paradigmas (KuHN, T.); orientaciones teóricas (KAPLAN, D. y MANNERS, R., 1968); estrategias de investigación (HARRIS, M., 1968) o sencillamente escuelas o corrientes. La homogeneidad que aparentan cubrir esas denominaciones oculta, por cierto, una gran heterogeneidad en sus contenidos y operatorias. Por ejemplo, la explicación en Antropología es evaluada de muy diversas maneras $-\mathrm{y}$ por tanto conducida por muy diversas metodologías-, según se apunte a establecer relaciones de causalidad de tipo determinístico (STEward, J.; White, L.; Harris, M. e.g.) o más de tipo comprensivo, en la búsqueda del sentido subyacente en el comportamiento humano, asunto que compromete a toda la tradición boasiana de la Antropología cultural, sus discípulos relativistas culturales y a la influencia de Evans-Pritchard en Inglaterra.

Nosotros preferiríamos utilizar la denominación perspectivas antropológicas para referirnos a la operatoria y campos de interés del evolucionismo, difusionismo, funcionalismo, configuracionalismo, cognoscitivismo, etc. El concepto perspectiva ilumina acerca de la posibilidad que investigadores y estudios conducidos por diferentes supuestos no entreguen resultados coincidentes, dados los diversos ángulos de la realidad que destacan. Nuestro enfoque con respecto a este problema está implícito, desde hace largo tiempo, en la contestación que dio R. Redfield a las ácidas observaciones de $\mathrm{O}$. Lewis a su estudio en Tepoztlan. Por cierto, el alcance de las explicaciones y los criterios de intersubjetividad sólo resultan ser cabalmente atendidos al interior de la perspectiva seleccionada.

Como quiera que se las denomine, las perspectivas antropológicas compiten entre sí, su adecuación estaría dada por la claridad y el tipo de diferenciación que proporcionan respecto de los ámbitos culturales que nos interesan. Por cierto, las inclinaciones del investigador y las 
características inherentes a su objeto de estudio -reales o imaginarias-- juegan un importante papel en la selección de una u otra perspectiva. Es en este sentido que afirmamos que no existe la teoria antropológica; hasta podría no ser deseable su existencia, pues ella -sospechamos- se sustentaria, de uno u otro modo, en una visión dogmática de la realidad, sustentada, como otras veces ha ocurrido, por intereses ajenos a la ciencia misma.

A continuación proseguiremos nuestra exposición revisando un conjunto de perspectivas antropológicas, las cuales se caracterizan por dar primacía a los aspectos ideacionales de la actividad humana, es decir, a lo que corrientemente se denomina cultura no-material, ideofacturas, mundo simbólico, ideología, representaciones colectivas, etc. Posteriormente desarrollaremos más en extenso una importante variante de ellas: la perspectiva cognitiva, especialmente la versión del antropólogo estadounidense Ward Goodenough.

\section{Perspectivas ideacionales de la Cultura y sus relaciones Con la Antropología Cognitiva}

En la perspectiva cognitiva desarrollada y actualmente en boga en la Antropología sociocultural, se aplica una definición de la cultura en que ésta se restringe al nivel ideacional de la vida humana. Así, según W. Goodenough, la cultura "... es, más bien, la forma que tienen las cosas en las mentes de la población y los modelos de las mismas para percibirlas, relacionarlas e interpretarlas" (1975, p. 20). La cultura, de acuerdo a esa interpretación, queda asentada en los individuos, específicamente en sus ordenaciones cognitivas más preciadas y recurridas; no en vano este enfoque es denominado también como la etnociencia. En esa perspectiva se inscribe un contingente bastante importante de antropólogos. De ellos se destacan S. Tyler, Ch., Frake, W. Sturtevant, H. Conklin, D. Metzger, L. Lounbury, A. F. Wallace, A. K. Romney y R. D'Andrade entre otros nombres.

La Antropología cognitiva se relaciona estrechamente con la perspectiva denominada cultura-y-personalidad, ambas, en un sentido amplio, son sicologizantes, en lo que respecta a su tratamiento de la cultura y al mismo tiempo compiten en idéntico ámbito del desarrollo de la Antropología académica, esto es, en la denominada Antropología cultural estadounidense y su área de influencia. El entronque inicial entre ambas perspectivas ocurre a partir de las investigaciones antropológicas que se enmarcan en la tradición boasiana y, particularmente, para el caso de los cognoscitivistas, en la línea de estudios que se originaron en la vertiente lingüística de la Antropología y que dio lugar a la formulación de la famosa hipótesis de Sapir y Whorf, en la cual se sostenía que la realidad se modela y reconoce a través de las pautas del lenguaje que coresponden a una cultura. E. Sapir, antropó- 
logo y lingüista, discípulo de Bcas, es citado por representantes de ambas líneas como el pionero de sus perspectivas ${ }^{2}$.

Al igual que en el enfoque de cultura-y-personalidad, los antropólogos que adhieren a la perspectiva cognoscitiva resaltan el carácter de aprendido que tiene la tradición cultural en una sociedad; pero los conceptos de socialización y procesos enculturativos son escasamente aludidos; el orden cognitivo-cultural se da como un hecho. Es conveniente destacar que mientras los antropólogos cognitivos apuntan sus estudios más hacia las estructuras de la mente, especificamente sobre sus mecanismos y componentes perceptivos, los sicoculturalistas, sin ignorar aquellos aspectos, están más interesados en los sistemas de personalidad, especialmente de aquellos contenidos que contribuyen a explicar los patrones de comportamiento compartidos en grupos sociales y culturas. De la misma manera, la homogeneidad o la heterogeneidad cultural, el cambio o la estabilidad de las culturas, son estudiadas y analizadas desde distintos ángulos. El énfasis en las estructuras y procesos implicados en la percepción y la organización de la realidad, sitúa a los antropólogos cognitivistas en un plano eminentemente ideacional. Sus logros recaen, por tanto, en las luces que nos proporcionan para entender este importante campo de la vida humana en sociedad.

La preocupación por los fenómenos del lenguaje nos conduce a establecer el tipo de relación que podria existir entre la perspectiva estructuralista y la cognitiva.

Desde la visión levistrossiana del estructuralismo, condensada en su famiosa Antropologia Estructural (1968), pueden ser bosquejadas algunas importantes diferencias entre esa perspectiva y la que nos ocupa. El énfasis en el individuo y en las estrategias de investigación marcadamente inductivas, que constituyen un sello característico de los cognoscitivos en Antropología, están casi imperceptibles en el estructuralismo. Para C. Lévi-Strauss la cultura es una estructura de la cual los individuos no son del todo conscientes; en consecuencia, su estrategia de investigación es claramente deductiva, para lo cual se apoya en modelos lingü ísticos, matemáticos, biológicos y hasta cibernéticos. Sólo la nıecánica superficial de sus análisis tiende a ser similar para ambas perspectivas -identificación de rasgos relevantes, set de oposiciones, etc.--, pero los criterios que subyacen son diferentes: deductivos y etics para los estructuralistas y emics e inductivos en los investigadores cognoscitivistas.

En síntesis, las tres perspectivas antropológicas que hemos cotejado: cultura y personalidad, estructuralismo y cognoscitivismo, resaltan, con mayor o menor intensidad, aunque desde diversos ángulos,

\footnotetext{
${ }^{2}$ Una visión sintética del enfoque de cultura-y-personalidad puede ser encontrado en un artículo de C. Haefner, al cual, además, agradecemos sus comentarios sobre el presente ensayo. Nuestra gratitud se extiende al profesor M. Dannemann por sus valiosas sugerencias sobre la forma de expresar mejor nuestras ideas.
} 
por enfatizar el plano ideacional de la cultura. destacándose en ello los cognoscitivistas y los estructuralistas y, en alguna medida menor, los sicoculturalistas que persisten en su interés por el comportamiento. En las líneas que prosiguen examinaremos con mayor detenimiento la perspectiva antropológica cognoscitiva.

\section{El estudio de la CUltura desde la PERSPECTIV a COgNoscitiva}

La perspectiva antropológica de la cual es representante W. Goodenough es aquella que establece la primacía de las categorizaciones cognitivas por sobre la acción social, transformándose lo primero en recurso explicativo de la segundo. Si bien la relación propuesta ha sido escasamente demostrada en la investigación, como lo destaca y documenta reiteradamente $M$. Harris (1982), se insiste en ella al distinguir de una manera resuelta dos niveles $u$ órdenes distintos de la realidad cultural "... uno es el orden fenomenológico de los acontecimientos observados y las regularidades que exhiben [...] y el otro es [...] el orden ideacional que no es propiedad de la comunidad, sino de sus miembros [...] el orden ideacional, a diferencia del orden estadístico no es material, estando compuesto de formas ideales ..." (GooD ENOUGH, W., 1975a, p. 37) El orden ideacional estaría compuestu por “... las percepciones, los conceptos, las recetas y habilidades necesarias [...] las cosas que necesitan saber los individuos con el objeto de hacer cosas que cumplan las normas de sus compañeros" (Goodenough, W., 1975b, p. 190).

La concepción de cultura que se expuso se asemeja bastante a una sicoculturalista, en la que ésta es concebida como diseños de vida que orientan el comportamiento. Sin embargo, los contenidos culturales presentes en las mentes de los individuos son, en la operatoria de los antropólogos cognoscitivos, aislados del comportamiento real y a través de ese procedimiento nos sumergimos en una incertidumbre entre lo que los informantes dicen -o dicen pensar- y lo que realmente hacen. Esta estrategia de investigación se fundamenta en establecer una relación entre la manera mediante la cual el ambiente es experimentado por los individuos, lo que nos remite a sus sistemas cognitivos, y el repertorio lingüistico que permite hablar sobre ese ambiente y experiencia, es decir, su sistema terminológico ${ }^{3}$.

Al ser entendida la cultura como un conjunto de posibilidades de acciones --expectativas--, o conjuntos de reglas y categorizaciones, se llega a concluir en aceptar la existencia de una suerte de gramática cultural, la cual, como todo conjunto de normas, es frecuentemente

\footnotetext{
${ }^{3}$ En estas últimas décadas también la sociología se ha visto fuertemente influida por estas perspectivas, pero su resolución metodológica ha escapado de la vertiente lingüística al adherirse a los patrones propuestos por la fenomenología, incorporada a la tradición académica estadounidense por el filósofo A. Schutz e impulsada por Harold Garfinkel bajo la denominación de etnometodología.
} 
trasgredida o reinterpretada y como tal no es deducible del comportamiento. En concreto, la cultura, desde esta perspectiva, no se extrae de la acción humana observable, sino que de las ideas y teorías, tanto implícitas como explícitas, que tienen los partícipes de un grupo acerca de cuáles son las reglas que regulan su comportamiento, "la cultura proporciona un conjunto de expectativas referentes a qué clases de comportamientos son adecuados en determinadas situaciones..." (r. 194) ${ }^{4}$.

Tal como es enunciado por el propio Goodenough, existiría un paralelismo evidente entre el lenguaje y la cultura. Tanto la cultura como el lenguaje son concebidos como sistemas de normas de comportamiento -en un caso lingüístico y en el otro social-- y como principios para poner en orden tal comportamiento. Lo anterior tiene importantes implicaciones. Por ejemplo, al reconocerse los sistemas cognitivos en su expresión lingüística puede suponerse que toda la estructura cognitiva está sometida a la condición que le impone su medio de expresión, y siendo los fenómenos lingüísticos mejor conocidos se tiende a aplicar su modelo como si fuera una teoría de la cultura, cuando en realidad el lenguaje en sí es un fenómeno estrictamente cultural y no lo contrario. Estamos en presencia, por lo tanto, de un reduccionismo.

Al nivel de la investigación, la Antropología cognitiva privilegia al instrumental metodológico y técnico que nos permite explorar más directamente los denominados mundos subjetivos. Tanto ha contribuido al desarrollo de los métodos cualitativos, que la perspectiva cognoscitiva es absorbida casi completamente por su enfoque metodológico. No es extraño, por tanto, que esta perspectiva sea conocida también bajo el nombre de la nueva etnografía. Los nuevos etnógrafos serían estudiosos que, desde una perspectiva cognoscitiva y emics y apoyados por un modelo lingüístico, analizan las distinciones que forman la base de la operatoria ideal en un sistema sociocultural. Por cierto, estamos en presencia de un tipo de reduccionismo que se apoya, como antes lo señaláramos, en una supuesta relación entre lo cognitivo y lo terminológico, por un lado, y los patrones de comportamiento real, por el otro.

Cualquiera que sea el fenómeno cultural que se pretende estudiar, la información debe ser buscada en la mente de los miembros del grupo que nos interesa. En este sentido, el problema clave de los antropólogos cognoscitivos consiste en lograr desarrollar una metodología adecuada que permita discernir cómo los individuos reconstruyen su ambiente sin falsificar la manera con que ellos dicen hacerlo. Todo esto implica un arduo trabajo de campo y un papel vital lo juegan los informantes. El procedimiento es más o menos como sigue: se pide a los informantes diferenciar - de acuerdo a sus propios

\footnotetext{
${ }^{4}$ Una perspectiva relativamente similar había sido propuesta por A.F.C. Wallace en un clásico sobre Cultura-y-Personalidad (pp. 42-57).
} 
patrones- y luego clasificar algún aspecto de la realidad, sobre la base de esa información; posteriormente, se detcrminan dominios semánticos, se construyen series de contrastes y finalmente taxonomias. Este procedimiento es eminentemente cualitativo, implica una cálida fluidez de relaciones entre el informante y el investigador, un conocimiento de la lengua y el habla de la comunidad y la aplicación de una técnica de investigación denominada controlled eliciting, la cual es algo más que una conversación en la que se estimula el tratamiento de determinados tópicos, los cuales se registran tal cual fueron enunciados.

Scbre la información recogida se aplican instrumentos de análisis tales como el análisis componencial ${ }^{5}$, el análisis lógico semántico y en algunos casos procedimientos estadísticos. Lo que permite objetivar la información está justamente en el hecho de que las distinciones y categorizaciones de la realidad que tienen los informantes, como asimismo sus relaciones y normas que las rigen, se exploran en un nivel terminológico. Sin embargo, las dificultades para seleccionar informantes a través de muestreos aporta una seria crítica en torno a la representatividad de la información analizada $y$, naturalmente, a su validez y confiabilidad, especialmente cuando se trata de establecer algún tipo de generalizaciones que comprometan a una comunidad o a un sistema sociocultural completo. El procedimiento más aplicado, esto es, el análisis componencial, condiciona a esta perspectiva antropológica cognoscitivista a remitirse a dominios muy restringidos de la cultura, pues, como señala Sturtevant, "la descripción etnocientífica completa de una cultura requeriría de muchos miles de páginas, publicadas después de muchos años de trabajo de campo intensivo, basado en los métodos etnográficos más completos y avanzados que disponemos hasta ahora" (p. 130), en otras palabras: es impracticable para una Antropología en que primen los criterios de integralidad y holísticos, entendidos de una manera clásica. Muchos observadores, críticos de los logros de la Antropología cognitiva, indican que esta perspectiva solamente tiene éxito en ámbitos muy particulares de la cultura, especialmente en el campo del parentesco, la medicina, la visión de colores y en las clasificaciones zoológicas y botánicas. Todo esto lleva a algunos antropólogos a afirmar, de una manera infundada, a nuestro parecer, que la perspectiva cognoscitiva "... se muestra totalmente falta de adecuación, salvo en lo referente a las terminologias en culturas ágrafas, omitiendo gran parte de las áreas de la experiencia humana que son de interés para los antropólogos" (Ross I y O'Higgins, p. 120).

Como se desprende de nuestra exposición, la Antropología cognitiva, consecuente con su definición ideacional de la cultura, traslada su

\footnotetext{
${ }^{5} \mathrm{El}$ mismo Goodenough fue designado por los editores de la Enciclopedia Internacional de las Ciencias Sociales para exponer el acápite correspondiente al análisis componencial (1968, vol. 3, pp. 186-192).
} 
problema epistemológico a sus procedimientos metodológicos, específicamente en el rescate de las perspectivas subjetivas, para lo cual se apoya en la denominada estrategia emics de la investigación cultural.

La estrategia investigativa emics se opone a una modalidad tradicional denominada etics; ambas denominaciones provienen de la lingüística y fueron enunciadas, en sus actuales significados y usos, por primera vez, por el antropólogo y lingüista Kenneth Pike a mediados de la década del 50. Los términos en cuestión provienen de la distinción entre el dominio de la fonética y el de la fonémica. La fonética trata de condiciones medibles exteriormente, incluso a nivel de factores físicos. Una descripción fonética en la lingüística consiste en analizar el lenguaje hablado de acuerdo, por ejemplo, a sus dimensiones de articulación. El código que se desprende de este nivel de análisis puede ser aplicado en cualquier lenguaje. Los hablantes, por cierto, no requieren ser conscientes de las discriminaciones que contribuyen a explicitar. La fonémica, por el contrario, trata de los fonemas, es decir, aquellas distinciones de sonidos que tienen algún significado para sus hablantes. Mientras la observación etics se dirige a detectar distinciones, la emics se proyecta en observar cómo esas distinciones son observadas por los miembros de un grupo. En el lenguaje cibernético se hablaría en este último caso de una observación de segundo orden.

De esta distinción linguística entre la fonética y fonémica se sirven los antropólogos cognoscitivos para diferenciar entre dos estrategias en la investigación de la cultura: la que se dirige a la visión interna de las culturas, y la observación de las culturas de acuerdo a categorías externas a ellas. Mientras que un investigador se apoya en principios cientificistas -etics- y se afana en comprobar o refutar hipótesis interculturales, el investigador emics intenta reproducir la realidad cultural desde el punto de vista de sus informantes. En sintesis, el enfoque emics, que caracteriza a la Antropología cognoscitiva, intenta describir y comprender fenómenos culturales rescatados del repertorio conceptual cotidiano de sus partícipes.

Corrientemente se acusa a los antropólogos cognoscitivistas de ser ardientes defensores de un enfoque extremadamente particularista de las culturas y tributarios del relativismo cultural a ultranza; sin embargo, ello no es totalmente correcto. Al menos en la versión de Goodenough, estos antropólogos aspiran a elaborar también proposiciones generales y desarrollar hipótesis de validez intercultural, y de tal manera "... poder describir los componentes émicos originarios de cualquier sistema de comportamiento ..." (1975b, p. 168). El procedimiento a seguir es claramente inductivo, se trata de hacer el máximo de descripciones culturales émicas, en todos los dominios posibles y extraer posteriormente de ellas regularidades discriminacionesuniversalmente válidas. Una de las metas de la Antropología cognoscitiva sería, por lo tanto, construir un código intercultural, conciliándose con ello con las metas e intereses de la Antropología clásica. 
En el intertanto, uno de los más importantes aportes de la perspectiva cognoscitiva en Antropología ha sido el desarrollo de los procedimientos metodológicos que bajo su impulso se han promovido $y$, sin duda, su acertada crítica hacia una tradicional modalidad de investigación antropológica: los denominados cross cultural survey, en los cuales se aplican aproximaciones seudoobjetivas y que de hecho son aproximaciones emics correspondientes a una determinada cultura, usualmente la estadounidense y sus estratos medios urbanos.

Indudablemente las críticas hacia los estudios comparativos e interculturales tienen bastante peso, pero los cognoscitivos no están libres de los problemas que esgrimen contra las otras perspectivas: ¿es posible un aislamiento total de lo etics en la estrategia emics? Los etnocientistas, quiéranlo o no, interfieren en la información cultural-cognitiva desde el mismo momento en que seleccionan un dominio semántico. Para contrarrestar estas desviaciones, algunos antropólogos, guiados por principios emicistas totales, solamente han llegado a trivialidades; es el caso, por ejemplo, de un estudio en el que se construyó una taxonomía de algo para comer originada de observaciones en un Burger Inn estadounidense y en la cual se llegan a clasificaciones que bien hubieran sido posibles de extraer de la lectura de la lista de precios (Frake, C. ). No en vano, A. Burling criticaba a los etnocientistas ortodoxos duramente señalando que el análisis componencial y toda su periferia metodológica no era más que un montón de bromas y que no proporcionaban nada sustantivo a la Antropología; no menos ácidas son las observaciones que hace $M$. Harris al respecto (1968).

Es evidente que existen fuertes limitaciones para el desarrollo de la perspectiva antropológica que comentamos, interrogantes tales como: ¿qué parte de la cultura está realmente contenida en las mentes de los individuos?, o ¿qué tan culturales son las categorías cognitivas? ${ }^{6}$. Al margen de lo anterior otras inquietantes preguntas surgen rápidamente: ¿cuál es la relación que hay entre el sistema cognitivo y el comportamiento real?; al respecto existe siempre el problema del nivel de explicación que se persigue. El antropólogo no solamente describe, sino que su interés es la explicación de fenómenos culturales. Al parecer, muchas ceremonias rituales y de otro tipo pueden ser excelentemente descritas émicamente, pero, en el fondo, seguirían sin explicarse si no se recurre a otro tipo de procedimientos. El problema de las relaciones causales permanece también sin resolverse, por ejemplo, permanece sin discutirse el peso de los factores adaptativos y cognitivos en lo que respecta a la explicación y curso de nuestro comportamiento.

\footnotetext{
${ }^{6}$ Este problema ha sido debatido no solamente desde la postura piagetana en la sicología del desarrollo, sino, además, por el mismo C. Lévi-Strauss. En ambos no se descartan los aspectos biológico-estructurales involucrados en la cognición.
} 
Evidentemente existen, aparte de lo ya mencionado, importantes vacíos e interrogantes no resueltos en la Antropología cognoscitiva, incluso sus seguidores las afrontan de una manera dispersa; sin embargo, y hay que recalcarlo, la perspectiva cognoscitiva acerca de la cultura tiene bastantes puntos de interés y resulta bastante útil para clarificar distinciones que en otros enfoques son dejados de lado o ignorados. En efecto, las ideas y relaciones contenidas en la Antropología cognitiva nos permiten, a través de las nuevas experiencias de diferenciación que proporcionan sus conceptos, abordar parte importante de la complejidad que se implica en cualquier segmento de la realidad sociocultural.

Por último, resulta de interés para todo antropólogo reconocer las formas y algunos de los contenidos del pensamiento de los partícipes de una cultura, independientemente de si estos temas son o no considerados como lo sustancial o como único objeto de estudio propiamente antropológico. En todo caso, la competencia entre las diversas perspectivas antropológicas que hoy están en escena, tendrá siempre como resultado acrecentar nuestras posibilidades para generar nuevos y mejores medios para el análisis de la realidad cultural y para su transformación consciente.

\section{Bibliografía}

Boas, F. "The limitations of the comparative method of anthropology", Science. 18, pp. 901-909, 1896.

Burling, A. "Ccgnition and componential analysis: God's truth or hocus-pocus". American Anthropologist, 66. pp. 20-28, 1964.

Frake, C.O. "Die ethnographische Erforschung kognitiver Systeme", Arbeitsgruppe Bielefelder Soziologen (eds.), Bielefeld, 1973.

Garfinkel, H. Studies in Ethnomethodology, New Jersey, Prentice-Hall, Inc., 1967.

Goodenough, w. "Componential Analysis", en Encyclopedia of the Social Siences, vol. 3 , pp. 186-192, 1968.

Goodenough, W. "Introducción", en La Antropologia como ciencia, José R. Llobera (ed.), Barcelona, Ed. Anagrama, pp. 25-45, 1975 a.

Goodenough, W. "Cultura, lenguaje y sociedad", en El concepto de cultura: textos fundamentales, J.S. Kahn (comp.), Barcelona, Ed. Anagrama, pp. $157-248,1975 b$.

Haefner, C. "Algunos alcances sobre el desarrollo y consolidación científica de la Antropología Psicológica", Revista Chilena de Antropologia, No 5, pp. 55-65, 1986.

Harris, M. The Rise of Anthropological Theory. A History of Theories of Culture, New York, Thomas Crowell Company (ed.), 1968.

Harris, M. El materialismo cultural, Madrid, Ed. Alianza, 1982.

Kaplan, D. y Manners R. "Notes on Theory and Non-Theory in Anthropology", en Theory in Anthropology: a Sourcebook, D. Kaplan et al., Aldine Publishing Company, 1968. 
Kaplan, D. y Manners, R. Introducción critica a la teoria antropológica, México, Ed. Nueva Imagen, 1981.

Kuhn, T. La estructura de las revoluciones cientificus, México, Fondo de Cultura Económica, 1971.

Lévi-Strauss, C. Antropologia Estructural, Buenos Aires, Ed. Eudeba, 1968.

Lewis, O. Tepoztlan, un pueblo de México, Ed. Joaquín Mortiz-México, 1968.

Merton, R. Teoria y Estructuras sociales, México, Fondo de Cultura Económica, 1968.

Pike, K. "Puntos de vista éticos y émicos para la descripción de la conducta", en Comunicación y Cultura, A. Smith (comp.), Buenos Aires, Ed. Nueva Visión, 1972.

Rossi, I. y O'Higgins, E. Theories of Culture and Anthropological Methods, New York, Bergin Publishers, 1980.

Sturtevant, W.C. "Studies in Ethnoscience", American Anthropologist, pp. 99-131, $1966(2)$.

Steward, J. Theory of Culture Change, University of Illinois Press, 1955.

Tyler, S. Cognitive Anthropology, New York, Holt, Rinehart and Wiston, 1969.

Wallace, A.F.C. Cultura y Personalidad, Buenos Aires, Ed. Paidós, 1963.

White, L. La Ciencia de la Cultura, Ed. Paidós, Buenos Aires, 1949. 